\title{
A HYBRID CONTINUOUS-DISCRETE APPROACH TO LARGE DISCRETE STRUCTURAL OPTIMIZATION PROBLEMS
}

\author{
B. Btachowski ${ }^{1}$, W. Gutkowski ${ }^{2}$ \\ ${ }^{1}$ Institute of Fundamental Technological Research, Warsaw, Poland \\ ${ }^{2}$ Institute for Mechanized Construction and Rock Mining, Warsaw, Poland
}

\section{Introduction}

Engineering systems are often composed of prefabricated elements, parameters of which are listed in professional catalogues. In the case of structural design, the catalogue is in the form of a list of available rolled profiles. The minimum weight structural design consist then in assigning to all structural members elements from the catalogue, assuring fulfilments of imposed constraints. Such a process is known as Discrete Structural Optimization (DSO). The difficulties arising in DSO come from relatively large numbers of combinations between obtained from both numbers of structural members and available parameters. These numbers can reach values of more than $10^{10}$. This makes impossible to find a minimum just from a direct enumeration.

The first attempts to solve Discrete Structural Optimization were made in 1960s and 1970s. In the beginning of 1980s several stochastic approaches were applied in DSO. Most known are: Genetic Algorithm; Simulated Annealing; Evolutionary Optimization. Recently, other stochastic methods based on Particle Swarm optimization by Kitayama et al.[1] and Harmony Search by Lee et al. [2] has been applied to DSO.

The concept of controlled discrete optimization was proposed in earlier authors papers. Gutkowski proposed a controlled mutation consisting in verification of stresses at each generation of the evolutionary optimization algorithm. Also, by Gutkowski et al.[3], a control of stresses was applied in an algorithm in which redundant material is removed in elements with least stresses. This approach was then applied by Guerlement et al. [4] DSO, taking in to account EC codes.

In this study, the idea of removing redundant material is enhanced by combining the continuous and discrete solutions. At the end of the paper, several numerical examples, with numbers of combinations up to $30^{8}$ are presented and their effectiveness is validated.

\section{Formulation of the problem}

The structure under consideration is of a given topology and composed of $j_{o}$ elements, which are taken from a list of available parameters as: thickness of a metal sheet $h$, cross section areas (CSA) $A$ and/or moments of inertia $I$ of a beam. Each $j$-th member having CSA $A_{j}$ is made of linear elastic material. Small displacements and stresses within elastic range are assumed for the whole structure. The structure is subjected to $q_{0}$ multiple static loads.

The governing equations, and in the same time equality constraints, for the problem, applying Finite Element Method (FEM), are:

$$
\begin{aligned}
& \mathbf{K} \mathbf{u}_{q}-\mathbf{Q}_{q}=\mathbf{0} \text { where } q=1 ; 2 \ldots . q_{0} \\
& \mathbf{K} \text {-stiffness matrix, } \mathbf{u}_{q} \text { - displacement, } \mathbf{Q}_{q} \text { - under } q \text { load }
\end{aligned}
$$

Inequality constraints, are imposed on:

- the largest and smallest values of listed parameters as $A_{\min } \leq A \leq A_{\max }$

- the maximum stresses and displacements $\sigma_{0}, u_{0}$

\section{The outline of the hybrid method}

A design of a structure (truss, frame, plates with reinforcements) has to be accomplished from a given catalogue of prefabricated elements, say rolled profiles. The design starts by finding the continuous structural optimization problem for the discussed structure, under given load and 
subjected to assumed constraints. Next, a catalogue, separately for each structural member is constructed. Two parameters from the catalogue are assigned to the member. One, larger than the continuous value and one smaller. Now, a discrete solution removing redundant material is obtained. The second step consists in enlarging catalogues for all members to two larger, and two smaller values from the obtained discrete solution in the first step. Again, the discrete solution for such a set of values is obtained. The procedure of enlarging the catalogue is ended when in two successive steps the discrete solution are the same.

\section{Numerical examples}

In order to illustrate applicability of the method, a 25 transmission tower with $30^{8}$ combinations, was analyzed. After calculations, it was observed that presented algorithm requires 100 FEM analyses, while for the same structure Evolutionary Optimization needs $10^{4} \mathrm{FEM}$ analyses.

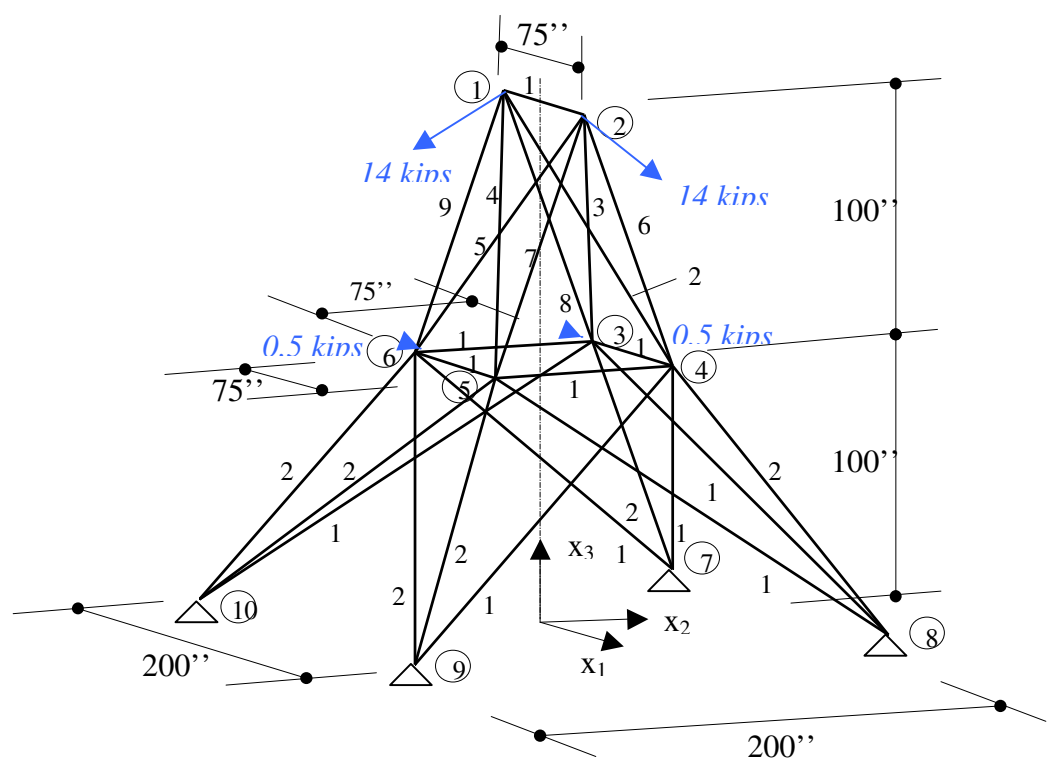

\section{References}

[1] Kitayama, S., Arakawa, M., Yamazaki, K., Penality function approach for the mixed discrete nonlinear problems by particle swarm optimization, Struct Multidisc Optim, 2006, 32(3) 191-202.

[2] Lee, K.S., Geem, Z.W., Lee, S., Bae, K.W., The harmony search heuristic algorithm for discrete structural optimization, Engineering Optimization, 2005, 37(7), 663-684.

[3] Gutkowski, W., Bauer, J. and Zawidzka, J., An effective method for discrete structural optimization. Engineering Computations, 2000, 17(4), 417-426.

[4] Guerlement, G., Gutkowski, W., Targowski, R., Zawidzka, J., Zawidzki, J., Discrete minimum weight design of steel structures using EC3 code, Struct Multidisc Optim, 2001 22, $322-327$. 